\title{
Interpretation and Utility of the Moments of Small-Angle X-Ray Scattering Distributions
}

\author{
Peter Modregger, ${ }^{1, *}$ Matias Kagias, ${ }^{2,3}$ Sarah C. Irvine, ${ }^{4}$ Rolf Brönnimann, ${ }^{5}$ \\ Konstantins Jefimovs, ${ }^{2,3}$ Marco Endrizzi, ${ }^{1}$ and Alessandro Olivo ${ }^{1}$ \\ ${ }^{1}$ Department of Medical Physics and Bioengineering, University College London, Gower Street, WCIE 6BT London, United Kingdom \\ ${ }^{2}$ Swiss Light Source, Paul Scherrer Institut, 5232 Villigen, Switzerland \\ ${ }^{3}$ Institute for Biomedical Engineering, UZH/ETH Zürich, 8092 Zürich, Switzerland \\ ${ }^{4}$ Diamond Light Source, Harwell Science and Innovation Campus, Didcot, OX11 ODE Oxfordshire, United Kingdom \\ ${ }^{5}$ Empa, Swiss Federal Laboratories for Materials Science and Technology, Reliability Science and Technology, \\ Überlandstrasse 129, 8600 Dübendorf, Switzerland
}

(Received 2 June 2016; revised manuscript received 7 April 2017; published 30 June 2017)

\begin{abstract}
Small angle x-ray scattering has been proven to be a valuable method for accessing structural information below the spatial resolution limit implied by direct imaging. Here, we theoretically derive the relation that links the subpixel differential phase signal provided by the sample to the moments of scattering distributions accessible by refraction sensitive $\mathrm{x}$-ray imaging techniques. As an important special case we explain the scatter or dark-field contrast in terms of the sample's phase signal. Further, we establish that, for binary phase objects, the $n$th moment scales with the difference of the refractive index decrement to the power of $n$. Finally, we experimentally demonstrate the utility of the moments by quantitatively determining the particle sizes of a range of powders with a laboratory-based setup.
\end{abstract}

DOI: 10.1103/PhysRevLett.118.265501

Small angle $\mathrm{x}$-ray scattering (SAXS) provides access to morphological information about the sample on nanometer to micrometer scales without the need to resolve the structures. Several $\mathrm{x}$-ray imaging techniques are sensitive to small angular deviations in the $\mathrm{x}$-ray direction allowing for the retrieval of the SAXS signal, including coherent $\mathrm{x}$-ray scattering (CXS) [1,2], analyzer-based imaging (ABI) [3,4], grating interferometry (GI) [5-7] and edgeillumination (EI) [8,9]. Examples for the utilization of SAXS just from the field of biomedical imaging range from cancer detection [10-13] to bone structure determination [14-16] to the diagnosis of pulmonary emphysema [17-19]. The ability to differentiate between subpixel structures opens the intriguing possibility of increasing pixel sizes and decreasing dose, while maintaining access to the desired sample information.

Most of these investigations focus on three contrast modalities: absorption, refraction, and scatter width (or dark-field contrast in the case of GI), where the latter is experimentally observed through a broadening of the techniques' response function (or reduction of visibility in case of GI). The three modalities can be viewed as parameters describing the angular resolved scattering distribution $g(\alpha)$ with the scattering angle $\alpha$. The uncentralized moments of $g$ are given by [20]

Published by the American Physical Society under the terms of the Creative Commons Attribution 4.0 International license. Further distribution of this work must maintain attribution to the author(s) and the published article's title, journal citation, and DOI.

$$
M_{n}=\int d \alpha \alpha^{n} g(\alpha)
$$

with $n$ an integer denoting the order of the moment. It has been experimentally demonstrated that $M_{0}$ corresponds to absorption, $M_{1} / M_{0}$ to refraction, and $M_{2} / M_{0}$ to the scatter width $[3,7,19,21,22]$.

In the following, we will relate the moments $M_{n}$ directly to the phase signal provided by the sample $\phi(x)$. This will be done for two different models of scattering distributions. First, we will use the power spectrum of the complex wave amplitude as a model for the scattering distribution, which is a well-established fact for CXS and related techniques [2] and a reasonable approximation for GI and ABI [23-26]. Second, we will use the histogram over refraction angles as a model for the scattering distribution and we will argue that this applies for the incoherent illumination conditions utilized by EI. For simplicity's sake, we will limit the discussion to one dimensional sensitivity to refraction. Extension to the two dimensional case applicable to CXS is straightforward.

In terms of experimentally accessing the scattering distribution, the four $\mathrm{x}$-ray imaging techniques discussed here can be classified in two categories. In the first category the scattering distribution is directly provided by the detector signal, which is the case for CXS. In fact, it is well known that for CXS and related techniques the observable intensity at the detector $I(x)$ is directly proportional to the power spectrum of the complex wave field of the sample $D(x)$ [2], i.e.,

$$
I(x) \propto|\hat{D}(K x / z)|^{2},
$$




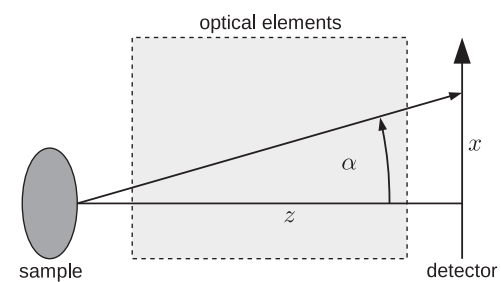

FIG. 1. Generic representation of an imaging system sensitive to small angle x-ray scattering. The shaded box represents optical elements for several methods: an analyzer crystal for ABI, two gratings for GI, two masks for EI, and absent optics (but large $z$ ) for CXS.

where $\hat{D}$ denotes the Fourier transform of $D(x), K$ is the wave number, and $z$ is the distance between the sample and detector (see Fig. 1). Please note that the shape of the incident beam can be accounted for by redefining the complex wave amplitude as $D(x)=D_{s}(x) D_{b}(x)$ with $D_{s}$ the contribution from the sample and $D_{b}$ the contribution from the beam shape, respectively.

Imaging techniques that experimentally utilize optical elements fall into the second category. Scanning one optical element (i.e., the analyzer crystal for ABI, a grating for GI, and a mask for EI) with and without the sample provides the method-specific response functions $s(\alpha)$ and $f(\alpha)$ for each detector pixel, respectively. For these techniques the scattering distribution $g(\alpha)$ appears in a convolution $[19,27,28]$.

$$
s(\alpha)=f(\alpha) \otimes g(\alpha),
$$

where $\otimes$ denotes the convolution operator. Thus, $g$ can be accessed from experimental data by deconvolution.

The width of $g(\alpha)$ (i.e., its 2 nd moment) corresponds to scatter strength, which is directly related to the so-called dark-field contrast [6] in GI [7]. Considerable investigations have been performed with the aim of acquiring a deep understanding of the dark-field contrast, which were mainly aimed at establishing a theoretical basis for tomographic reconstruction [28-32]. However, increasing theoretical and experimental evidence [23-25] suggests that $g$ can be reasonably approximated by the power spectrum of the sample, i.e.,

$$
g(\alpha) \propto|\hat{D}(\alpha K)|^{2} .
$$

Because of the similarity of provided contrasts in GI and ABI, we speculate that an analogous approximation should be valid for ABI. Thus, we will assume that the scattering distributions accessible by $\mathrm{CXS}, \mathrm{GI}$, and $\mathrm{ABI}$ are all proportional to the power spectrum of the sample. In contrast to CXS, GI, and ABI, which utilize coherent illumination conditions, EI employs incoherent illumination, which will allow us to further approximate $g$ later in this article. In order to distinguish these two cases we will label the scattering distributions as coherent and incoherent, respectively.

Using the small angle approximation for the scattering angle $\alpha=x / z$ in Eq. (2) leads to the coherent scattering distribution $g_{\mathrm{coh}}$

$$
g_{\mathrm{coh}}(\alpha)=K|\hat{D}(\alpha K)|^{2} .
$$

Here, we have normalized $g_{\text {coh }}$, so that the total detected intensity equals the transmission through the sample, i.e.,

$$
\begin{aligned}
\int d x|D(x)|^{2} & =\int d q|\hat{D}(q)|^{2} \\
& =K \int d \alpha|\hat{D}(\alpha K)|^{2}=\int d \alpha g_{\mathrm{coh}}(\alpha),
\end{aligned}
$$

where Parseval's theorem was used. The integrals extend over one pixel, which were assumed to be infinitely wide (i.e., limits: $\pm \infty$ ) corresponding to the subpixel origin of the contrasts and negligible pixel cross talk for the relevant imaging techniques (ABI and GI). The Fourier transform of $g_{\text {coh }}(\alpha)$ constitutes an autocorrelation

$$
\hat{g}_{\mathrm{coh}}(\beta)=\frac{1}{\sqrt{2 \pi}} \int d x D^{*}(x) D(\beta / K+x),
$$

where $D^{*}$ denotes the complex conjugate of $D$ and $\beta$ the variable reciprocal to $\alpha$. The moments of $g(\alpha)$ correspond to derivatives at $\beta=0$ in Fourier space [20]

$M_{n}=\left.\frac{\sqrt{2 \pi}}{(-i)^{n}} g_{\mathrm{coh}}^{(n)}(\beta)\right|_{\beta=0}=\frac{1}{(-i K)^{n}} \int d x D^{*}(x) D^{(n)}(x)$,

where the superscript $(n)$ denotes the $n$th derivative and the fact that

$$
\left.\frac{\partial^{n}}{\partial \beta^{n}} D(\beta / K+x)\right|_{\beta=0}=K^{-n} \frac{\partial^{n}}{\partial x^{n}} D(x)
$$

was exploited. Rewriting the complex wave amplitude as $D(x)=A(x) e^{i \phi(x)}$ and assuming that $A(x)$ is a slowly varying function, so that $D^{(n)}(x)=A(x)\left(\partial^{n} / \partial x^{n}\right) e^{i \phi(x)}$, leads to

$$
M_{n}=\frac{1}{(-i K)^{n}} \int d x A(x)^{2} e^{-i \phi(x)} \frac{\partial^{n}}{\partial x^{n}} e^{i \phi(x)} .
$$

Using $A(x)^{2}=t(x)$, with $t(x)$ the local transmission of the sample, provides intermediate results for the first five moments:

$$
\begin{aligned}
M_{0}= & \int d x t(x), \\
M_{1}= & -\frac{1}{K} \int d x t(x) \phi^{\prime}(x), \\
M_{2}= & \frac{1}{K^{2}} \int d x t(x)\left[\phi^{\prime}(x)^{2}-\underline{\left.i \phi^{\prime \prime}(x)\right]},\right. \\
M_{3}= & -\frac{1}{K^{3}} \int d x t(x)\left[\phi^{\prime}(x)^{3}\right. \\
& -\underline{\left.\phi^{\prime \prime \prime}(x)+3 i \phi^{\prime}(x) \phi^{\prime \prime}(x)\right]} \\
M_{4}= & \frac{1}{K^{4}} \int d x t(x)\left[\phi^{\prime}(x)^{4}-3 \phi^{\prime \prime}(x)^{2}-4 \phi^{\prime}(x) \phi^{\prime \prime \prime}(x)\right. \\
& +\underline{i \phi^{\prime \prime \prime \prime}(x)-6 i \phi^{\prime}(x) \phi^{\prime \prime}(x)} .
\end{aligned}
$$


For the underlined terms $h(x)$ a primitive of the form $H(x)=\phi^{(m)}(x)^{l}$ with two integers $m$ and $l$ can be found. Thus, partial integration can be applied, which leads to

$$
\int d x t(x) h(x)=\left.t(x) H(x)\right|_{-\infty} ^{\infty}-\int d x t^{\prime}(x) H(x)=0,
$$

because $t( \pm \infty)=0$ and $t^{\prime}(x) \equiv 0$ according to the above assumption of a slowly varying absorption signal. Therefore, the moments simplify to

$$
\begin{aligned}
M_{n}= & (-K)^{-n} \int d x t(x) \phi^{\prime}(x)^{n} \quad \text { for } n \leq 3, \\
M_{4}= & \frac{1}{K^{4}} \int d x t(x)\left[\phi^{\prime}(x)^{4}-3 \phi^{\prime \prime}(x)^{2}\right. \\
& \left.-4 \phi^{\prime}(x) \phi^{\prime \prime \prime}(x)\right] .
\end{aligned}
$$

Higher order moments can be retrieved by following similar lines of reasoning. Equations (16)-(17) establish a direct connection between the moments of coherent scattering distributions and the phase signal of the sample for CXS, ABI, and GI. For GI the dark-field signal $B$ [6] is related to the second moment by $M_{2}=-2 \ln B$ if a Gaussian scattering distribution is assumed [28]. Thus, Eq. (16) provides the relation between the differential phase signal and the dark-field contrast as

$$
B=\exp \left[-\frac{1}{2 K^{2}} \int d x t(x) \phi^{\prime}(x)^{2}\right] .
$$

An important special case for the complex wave function is given by a binary, pure phase object (i.e., a nonabsorbing sample composed of two homogeneous materials, where one material could be air). In this case the complex wave function can be written as

$$
D(x)=\exp [i \phi(x)]=\exp \left[i K \delta \int d x d(x)\right],
$$

with $d(x)$ the thickness of one material and $\delta$ the difference between the refractive index decrement of the two materials. By inserting Eq. (19) into Eqs. (16)-(17) it can easily be shown that the magnitude of the $n$th moment scales as

$$
M_{n} \propto \delta^{n} .
$$

Notably, $M_{2} \propto \delta^{2}$ holds true, which provides a scaling law for the scattering width.

We will now turn to the case of incoherent scattering distributions $g_{\text {inc }}$. As mentioned above, EI constitutes a noninterferometric method [33] utilizing incoherent illumination conditions $[34,35]$. The method-specific response function without the sample $f(\alpha)$ is called the illumination curve (IC). Introducing a wedge-shape sample into the beam provides a single refraction angle $\alpha_{s}$ and shifts the IC, which leads to $f\left(\alpha-\alpha_{s}\right)$. Since we can assume incoherent illumination conditions, the signals from several refraction angles simply add up according to their weight $g_{\text {inc }}\left(\alpha_{s}\right)$, which means the IC in the presence of a sample $s$ can be expressed as $s(\alpha)=\int d \alpha_{s} g_{\mathrm{inc}}\left(\alpha_{s}\right) f\left(\alpha-\alpha_{s}\right)=g_{\mathrm{inc}}(\alpha) \otimes f(\alpha)$,

where $\otimes$ denotes a convolution $[9,29]$. The weight of the contributing refraction angles $g_{\text {inc }}(\alpha)$ is given by their histogram [7]

$$
g_{\text {inc }}(\alpha)=\int d x \delta_{D}\left[-\frac{\phi^{\prime}(x)}{K}-\alpha\right],
$$

where $\delta_{D}$ is Dirac's $\delta$ function and $\alpha=-\phi^{\prime}(x) / K$ [36] was used. Once again we assume negligible pixel cross talk, so that the limits of the integral are $\pm \infty$. Equation (22) holds true for negligible absorption and nonunity transmission $t(x)$ can be accounted for by using $t(x)$ as an additional weighting function

$$
g_{\mathrm{inc}}(\alpha)=\int d x \delta_{D}\left[-\frac{\phi^{\prime}(x)}{K}-\alpha\right] t(x) .
$$

The relation of the moments of $g_{\text {inc }}$ to the differential phase signal $\phi^{\prime}(x)$ can now be derived by using Eqs. (1) and (23) and performing the integral with respect to $\alpha$

$$
\begin{aligned}
& M_{n}=\int d \alpha \alpha^{n} \int d x \delta_{D}\left[-\frac{\phi^{\prime}(x)}{K}-\alpha\right] t(x), \\
& M_{n}=(-K)^{-n} \int d x t(x) \phi^{\prime}(x)^{n} .
\end{aligned}
$$

Equation. (25) establishes the relation between the moments of the incoherent scattering distribution and the differential phase signal provided by the sample. This result is remarkable in two ways. First, the first four moments for $g_{\text {inc }}$ are identical to the ones for $g_{\text {coh }}$ [Eq. (16)]. Thus, the histogram of refraction angles constitutes a valid approximation for the power spectrum of the sample. Second, the introduction of a binary, pure phase object leads again to the scaling law $M_{n} \propto \delta^{n}$, which, therefore, holds true for both coherent and incoherent imaging techniques.

In addition to explaining the moments of scattering distributions in terms of the differential phase signal Eqs. (16)-(17) or Eq. (25) can be used for the rapid estimation of expected scattering characteristics for a given phase signal without the need for time intensive numerical simulations. This will be useful for optimizing experimental parameters for specific samples.

As a notable example for the application of the theory laid out so far, we will experimentally demonstrate the possibility for quantitative subpixel structural analysis. This will be done by relating the scattering moments of various powders measured by laboratory-based EI to a structure size parameter $\sigma$ of a model phase distribution $\phi(x)$, and comparing the results to the average particle sizes determined by synchrotron-based microtomography.

The laboratory-based experiment was carried out with the EI setup at University College London (London, UK). EI utilizes two apertured masks as optical elements in order to provide access to the SAXS signal. The presample mask shapes the incident radiation into small beamlets, which are distorted by the sample due to scattering. These distortions 
are converted into detectable intensity variations by the detector mask. A lateral scan of the presample mask with and without the sample provides the sample IC $s(\alpha)$ and the flat IC $f(\alpha)$ [see Eq. (3)] for each detector pixel, respectively.

The source was a Rigaku MM007 microfocus rotating anode with a Mo target operated at $25 \mathrm{~mA}$ current and $40 \mathrm{kVp}$ voltage. A Pixirad-2 photon counting detector (PIXIRAD Imaging Counters s.r.l., Pisa, Italy) with an isotropic pixel size of $62 \mu \mathrm{m}$ was used. Both masks were manufactured by laser cutting (EMPA, Dübendorf, Switzerland) into $150 \mu \mathrm{m}$ tungsten foil (Goodfellow, Huntingdon, UK). The sample mask featured a pitch of $98 \mu \mathrm{m}$ and openings of $8 \mu \mathrm{m}$, while the detector mask had a pitch of $122 \mu \mathrm{m}$ and apertures of $12 \mu \mathrm{m}$. In order to achieve a high sensitivity to the tails of the illumination curve, the masks had a column-skipped design and raw images were binned 4 times for subsequent data analysis resulting in an effective pixel size of $248 \mu \mathrm{m}$. The sample to detector distance was $z=0.4 \mathrm{~m}$ and the total setup length amounted to $2 \mathrm{~m}$.

Five different powders (i.e., talcum, icing sugar, flour, vestosint-1184, and powdered milk) were put into plastic sample holders with rectangular cross sections and imaged on 33 IC positions with $5 \mathrm{~s}$ exposure for both the sample $s(\alpha)$ and the flat IC $f(\alpha)$, which resulted in a total scan time of 10 min. Lucy-Richardson deconvolution was applied with 1000 iteration steps in order to retrieve a scattering distribution $g_{\text {inc }}$ for each pixel. Subsequent moment analysis of the scattering distributions [Eq. (1)] was then used to determine the kurtosis $\kappa$ according to

$$
\kappa=M_{4} / M_{2}^{2} .
$$

$\kappa$ constitutes a dimensionless measure of tail strength of a distribution [20]. Please note that the kurtosis is independent of $\delta$ and, thus, of the powder's specific material as can be seen by combining Eqs. (20) and (26). The experimentally determined kurtosis for each powder is indicated as a dashed horizontal line in Fig. 2. More details about the experimental implementation and data analysis can be found in Ref. [19].

We will show that a Gaussian-like function for the phase signal, given as

$$
\phi(x)=K \delta \exp \left(-x^{6} / 2 \sigma^{6}\right),
$$

constitutes a simple yet reliable model for the scattering properties of powders. The dependence of the kurtosis on the structure size parameter $\sigma$ was calculated according to Eq. (25), where the aperture of the presample mask was taken into account by using a rectangular function for the transmission $t(x)$. The choice of $x^{6} / \sigma^{6}$ over $x^{2} / \sigma^{2}$ in the exponent ensured that the range of the model kurtosis (Fig. 2) matched the experimentally determined range for the different powders, i.e., $\kappa \in(3.0,5.2)$. The model kurtosis shows a reversal of its dependency on $\sigma$ at the point where truncation effects of the aperture (i.e., $8 \mu \mathrm{m}$ ) become noticeable, and we used the knowledge of the powder's relative grain sizes in order to identify the part of the curve

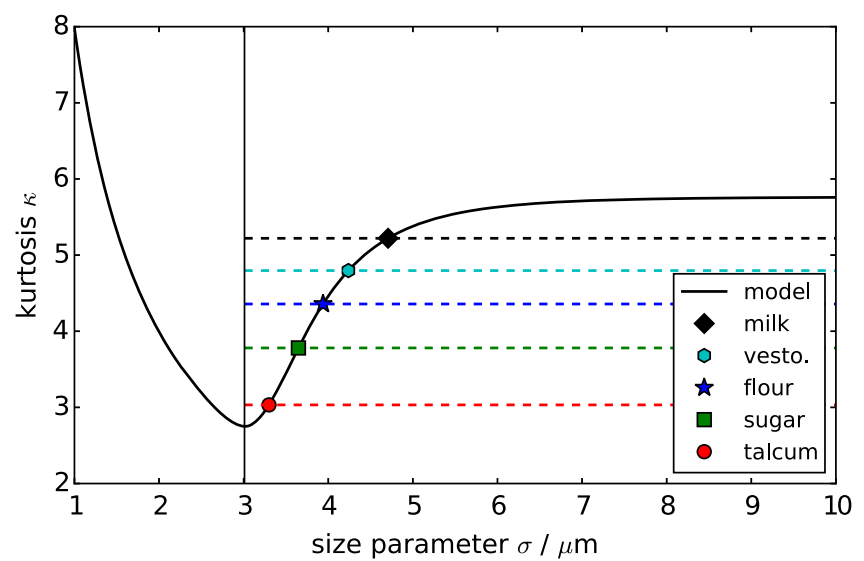

FIG. 2. Kurtosis of the model sample [Eq. (27)] as a function of the structure size parameter $\sigma$. Horizontal lines are the experimentally determined average kurtosises of different powders and the right-hand branch of the model's kurtosis was chosen for the determination of the powder's size parameters.

after the reversal point as the correct branch for the retrieval of the powders structure size parameter.

Synchrotron microtomography measurements were performed on the I13-2 Diamond-Manchester Imaging Beamline at Diamond Light Source (DLS)(Didcot, UK) in order to determine the particle size of the powders. Each powder was loosely scooped into separate inverted pipette tips, mounted individually onto a goniometer. The in-house detector system consisted of a $150 \mu \mathrm{m} \mathrm{CdWO} 4$ scintillator screen (Hilger Crystals Ltd) coupled to an optical microscope with $4 \mathrm{x}$ objective (Olympus UPLSAPO 4X) and a pco.edge 5.5 sCMOS camera (PCO AG, Kelheim, Germany), where a total optical magnification of eightfold yields an effective pixel size of $0.81 \mu \mathrm{m}$. Using pink beam, exposure times were $0.05 \mathrm{~s}$ with 3000 projections acquired over $180^{\circ}$ of rotation in $2.5 \mathrm{~min}$. Standard absorption-based tomographic reconstructions were performed using the tomo-recon pipeline at DLS, which makes use of the Diamond Clusters Grid engine for parallel-processed reconstruction based on the filtered back projection algorithm [37]. The mean particle size $r$ of the powders was determined by thresholding the reconstructed slices, labeling individual particles, calculating their cross-sectional area $A$, and then using $r=\sqrt{A / \pi}$. While averaging, particles with areas smaller than 100 voxels $^{2}$ were excluded in order to reduce the influence of noise. Representative reconstructed slices as well as final segmentations are available in the Supplemental Material [38].

Figure 3 compares the size parameter $\sigma$ of the powders extracted from the EI measurements to the mean particle sizes determined by microtomography. The very strong correlation $\left(R^{2}=0.92, p=0.009\right)$ clearly demonstrates a linear relationship between the model size parameter sigma and the actual particle sizes. The regression shown in Fig. 3 provides a calibration line that can be used to quantitatively determine unknown particle sizes of other powders and 


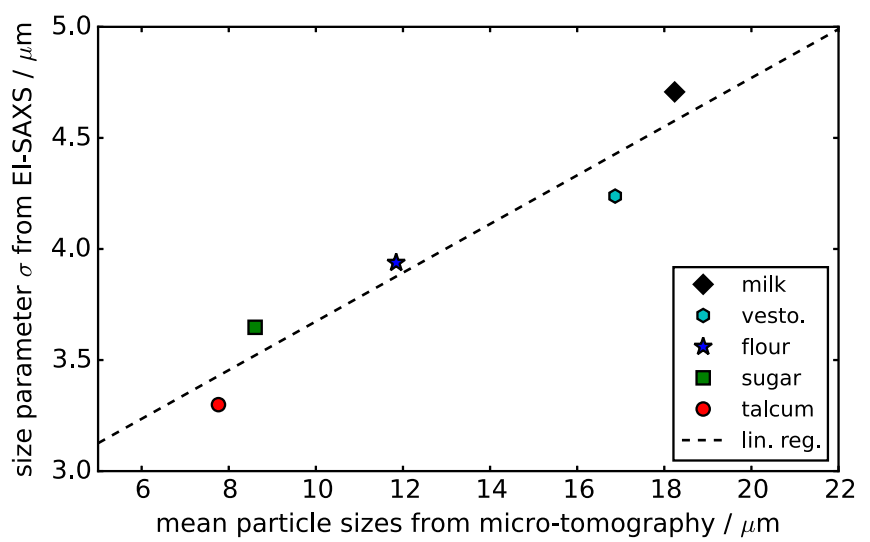

FIG. 3. Calibration for particle size determination by means of the moments of scattering distributions provided by EI-SAXS. The linear regression (dashed line) shows a very strong correlation $\left(R^{2}=0.92, p=0.009\right)$ between the size parameter $\sigma$ of the powders from EI-SAXS (Fig. 2) and their actual mean particle sizes as measured with microtomography.

similar samples through the proposed implementation of EI. By following an analogous approach, the proposed method should enable quantitative particle size determination with subpixel scattering contrasts also with other phase-based imaging methods (e.g., GI or ABI).

The robustness of this approach is made evident by the utilization of a laboratory-based x-ray source with a large spectrum as well as a wide range of powder materials and shapes that featured a significant spread of cross-sectional particle sizes [38], where other published approaches are limited to monodisperse microspheres [24,26]. Further, the strong correlation justifies the choice for the model sample [Eq. (27)] and the assumption of total incoherence for EI [Eq. (25)]. The result also indicates that the kurtosis of subpixel SAXS distributions can be interpreted as a measure of typical structure sizes of powderlike samples, which will be especially of interest in the context of detecting lung structure abnormalities [17,19].

In conclusion, we have theoretically derived the relation of the moments of coherent and incoherent scattering distributions to the differential phase signal generated by the sample. Further, we have demonstrated that the first four moments are identical for coherent and incoherent scattering distributions implying a close relationship in terms of accessible information between the corresponding techniques. In addition, we have shown that the magnitude of the $n$th moment scales with the refractive index decrement to the power of $n$ for binary phase objects. The developed theoretical framework can provide a basis for rapid estimation of the expected scattering characteristics and significantly adds to a deeper understanding of scatter contrast, which will support widespread applications of methods exploiting SAXS with refraction sensitive $\mathrm{x}$-ray imaging techniques. Finally, we utilized this framework in order to demonstrate a reliable experimental approach to determine the average particle size of a range of powders with subpixel scattering contrasts.
We thank Andrew Bodey from Diamond Light Source for assistance with the beam line set up. This project has received funding from the European Union's Horizon 2020 research and innovation programme under the Marie Skłodowska-Curie Grant Agreement No. 657621 and EPSRC Grant No. EP/I021884/1. M. Kagias is supported by the ERC Grant No. ERC-2012-StG 310005-PhaseX hold by Professor M. Stampanoni, ETH Zürich and Paul Scherrer Institute.

*p.modregger@ucl.ac.uk

[1] L. Feigin and D. Svergun, Structure Analysis by SmallAngle X-ray and Neutron Scattering (Plenum Press, New York, 1987).

[2] J. F. van der Veen, J. Phys. Condens. Matter 16, 5003 (2004).

[3] L. Rigon, H.-J. Besch, F. Arfelli, R. H. Menk, G. Heitner, and H. Plothow-Besch, J. Phys. D 36, A107 (2003).

[4] H. Suhonen, M. Fernández, A. Bravin, J. Keyriläinen, and P. Suortti, J. Synchrotron Radiat. 14, 512 (2007).

[5] C. David, B. Nöhammer, H. H. Solak, and E. Ziegler, Appl. Phys. Lett. 81, 3287 (2002).

[6] F. Pfeiffer, M. Bech, O. Bunk, P. Kraft, E. F. Eikenberry, and C. David, Nat. Mater. 7, 134 (2008).

[7] P. Modregger, F. Scattarella, B. R. Pinzer, C. David, R. Bellotti, and M. Stampanoni, Phys. Rev. Lett. 108, 048101 (2012).

[8] M. Endrizzi and A. Olivo, J. Phys. D 47, 505102 (2014).

[9] M. Endrizzi, P. C. Diemoz, T. P. Millard, J. Louise Jones, R. D. Speller, I. K. Robinson, and A. Olivo, Appl. Phys. Lett. 104, 024106 (2014).

[10] M. Fernández, J. Keyriläinen, R. Serimaa, M. Torkkeli, M.-L. Karjalainen-Lindsberg, M. Tenhunen, W. Thomlinson, V. Urban, and P. Suortti, Phys. Med. Biol. 47, 577 (2002).

[11] M. Stampanoni, Z. Wang, and T. Thüring, Investigative Radiology 46, 801 (2011).

[12] A. Olivo, S. Gkoumas, M. Endrizzi, C. K. Hagen, M. B. Szafraniec, P. C. Diemoz, P. R. T. Munro, K. Ignatyev, B. Johnson, J. A. Horrocks, S. J. Vinnicombe, J. L. Jones, and R. D. Speller, Med. Phys. 40, 090701 (2013).

[13] Z. Wang, N. Hauser, G. Singer, M. Trippel, R. A. KubikHuch, C. W. Schneider, and M. Stampanoni, Nat. Commun. 5, 3797 (2014).

[14] O. Paris, I. Zizak, H. Lichtenegger, P. Roschger, K. Klaushofer, and P. Fratzl, Cell. Mol. Biol. 46, 993 (2000).

[15] H. Wen, E. E. Bennett, M. M. Hegedus, and S. Rapacchi, Radiology 251, 910 (2009).

[16] G. Potdevin, A. Malecki, T. Biernath, M. Bech, T. H. Jensen, R. Feidenhans'l, I. Zanette, T. Weitkamp, J. Kenntner, J. Mohr, P. Roschger, M. Kerschnitzki, W. Wagermaier, K. Klaushofer, P. Fratzl, and F. Pfeiffer, Phys. Med. Biol. 57, 3451 (2012).

[17] A. Yaroshenko, F. G. Meinel, M. Bech, A. Tapfer, A. Velroyen, S. Schleede, S. D. Auweter, A. Bohla, A. Ö. Yildirim, K. Nikolaou, F. Bamberg, O. Eickelberg, M. F. Reiser, and F. Pfeiffer, Radiology 269, 427 (2013).

[18] F. G. Meinel, A. Yaroshenko, K. Hellbach, M. Bech, A. Velroyen, F. Bamberg, O. Eickelberg, K. Nikolaou, F. Maximilian, F. Pfeiffer, and O. Ali, Investigative Radiology 49, 653 (2014). 
[19] P. Modregger, T. P. Cremona, C. Benarafa, J. C. Schittny, A. Olivo, and M. Endrizzi, Sci. Rep. 6, 30940 (2016).

[20] F. James, Statistical Methods in Experimental Physics, 2nd ed. (World Scientific, Singapore, 2006).

[21] T. Weber, G. Pelzer, F. Bayer, F. Horn, J. Rieger, A. Ritter, A. Zang, J. Durst, G. Anton, and T. Michel, Opt. Express 21, 18011 (2013).

[22] O. Bunk, M. Bech, T. H. Jensen, R. Feidenhans'l, T. Binderup, A. Menzel, and F. Pfeiffer, New J. Phys. 11, 123016 (2009).

[23] W. Yashiro, Y. Terui, K. Kawabata, and A. Momose, Opt. Express 18, 16890 (2010).

[24] S. K. Lynch, V. Pai, J. Auxier, A. F. Stein, E. E. Bennett, C. K. Kemble, X. Xiao, W.-K. Lee, N. Y. Morgan, and H. Wen, Appl. Opt. 50, 4310 (2011).

[25] M. Strobl, Sci. Rep. 4, 7243 (2014).

[26] S. Gkoumas, P. Villanueva-Perez, Z. Wang, L. Romano, M. Abbis, and M. Stampanoni, Sci. Rep. 6, 35259 (2016).

[27] P. Modregger, D. Lübbert, P. Schäfer, and R. Köhler, Phys. Rev. B 74, 054107 (2006).

[28] Z. Wang, K.-J. Kang, Z.-F. Huang, and Z.-Q. Chen, Appl. Phys. Lett. 95, 094105 (2009).

[29] M. Bech, O. Bunk, T. Donath, R. Feidenhans'l, C. David, and F. Pfeiffer, Phys. Med. Biol. 55, 5529 (2010).
[30] G.-H. Chen, N. Bevins, J. Zambelli, and Z. Qi, Opt. Express 18, 12960 (2010).

[31] A. Malecki, G. Potdevin, and F. Pfeiffer, Europhys. Lett. 99, 48001 (2012).

[32] P. Modregger, M. Kagias, S. Peter, M. Abis, V. A. Guzenko, C. David, and M. Stampanoni, Phys. Rev. Lett. 113, 020801 (2014).

[33] A. Olivo, F. Arfelli, G. Cantatore, R. Longo, R. H. Menk, S. Pani, M. Prest, P. Poropat, L. Rigon, G. Tromba, E. Vallazza, and E. Castelli, Med. Phys. 28, 1610 (2001).

[34] P. R. T. Munro, K. Ignatyev, R. D. Speller, and A. Olivo, Opt. Express 18, 19681 (2010).

[35] M. Endrizzi, F. a. Vittoria, G. Kallon, D. Basta, P. C. Diemoz, A. Vincenzi, P. Delogu, R. Bellazzini, and A. Olivo, Opt. Express 23, 16473 (2015).

[36] S. W. Wilkins, T. E. Gureyev, D. Gao, A. Pogany, and A. W. Stevenson, Nature (London) 384, 335 (1996).

[37] R. C. Atwood, A. J. Bodey, S. W. T. Price, M. Basham, and M. Drakopoulos, Phil. Trans. R. Soc. A 373, 20140398 (2015).

[38] See Supplemental Material at http://link.aps.org/ supplemental/10.1103/PhysRevLett.118.265501 for reconstructed slices and results of segmentation of the powders as well as a table listing measured values for particle sizes, kurtosis, and structure size parameters. 